\title{
Improved Atmospheric Soundings and Error Estimates from Analysis of AIRS/AMSU Data
}

\author{
Joel Susskind \\ NASA Goddard Space Flight Center, Greenbelt, MD, USA 20771
}

\begin{abstract}
The AIRS Science Team Version 5.0 retrieval algorithm became operational at the Goddard DAAC in July 2007 generating near real-time products from analysis of AIRS/AMSU sounding data. This algorithm contains many significant theoretical advances over the AIRS Science Team Version 4.0 retrieval algorithm used previously. Three very significant developments of Version 5 are: 1) the development and implementation of an improved Radiative Transfer Algorithm (RTA) which allows for accurate treatment of non-Local Thermodynamic Equilibrium (non-LTE) effects on shortwave sounding channels; 2) the development of methodology to obtain very accurate case by case product error estimates which are in turn used for quality control; and 3) development of an accurate AIRS only cloud clearing and retrieval system. These theoretical improvements taken together enabled a new methodology to be developed which further improves soundings in partially cloudy conditions, without the need for microwave observations in the cloud clearing step as has been done previously. In this methodology, longwave $\mathrm{CO}_{2}$ channel observations in the spectral region $700 \mathrm{~cm}^{-1}$ to $750 \mathrm{~cm}^{-1}$ are used exclusively for cloud clearing purposes, while shortwave $\mathrm{CO}_{2}$ channels in the spectral region $2195 \mathrm{~cm}^{-1}$ to $2395 \mathrm{~cm}^{-1}$ are used for temperature sounding purposes. The new methodology for improved error estimates and their use in quality control is described briefly and results are shown indicative of their accuracy. Results are also shown of forecast impact experiments assimilating AIRS Version 5.0 retrieval products in the Goddard GEOS 5 Data Assimilation System using different quality control thresholds.
\end{abstract}

Keywords: High spectral resolution IR sounders, atmospheric sounding, satellite meteorology, new theoretical developments, improved forecast skill

\section{INTRODUCTION}

AIRS was launched on EOS Aqua on May 4, 2002, together with AMSU-A and HSB, to form a next generation polar orbiting infrared and microwave atmospheric sounding system. ${ }^{1}$ The primary products of AIRS/AMSU-A are twice daily global fields of atmospheric temperature-humidity profiles, ozone profiles, sea/land surface skin temperature, and cloud related parameters including OLR. Also included are the clear column radiances used to derive these products which are representative of the radiances AIRS would have seen if there were no clouds in the field of view. All products also have error estimates. The sounding goals of AIRS are to produce $1 \mathrm{~km}$ tropospheric layer mean temperatures with an rms error of $1 \mathrm{~K}$, and layer precipitable water with an rms error of 20 percent, in cases with up to 90 percent effective cloud cover. The products are designed for data assimilation purposes for the improvement of numerical weather prediction, as well as for the study of climate and meteorological processes. With regard to data assimilation, one can use either the products themselves or the clear column radiances from which the products were derived.

The basic theory used to analyze AIRS/AMSU/HSB data in the presence of clouds, called the at-launch algorithm, and that used in a post-launch algorithm, which differed only in the minor details from the at-launch algorithm, has been described previously ${ }^{2,3}$. The post-launch algorithm, referred to as AIRS Version $4,{ }^{3}$ has been used by the Goddard DAAC to analyze and distribute AIRS retrieval products. Susskind ${ }^{4}$ described the AIRS Version 5 retrieval algorithm, including an approach to provide AIRS soundings in partially cloudy conditions that does not require use of any data but AIRS observations. This new AIRS only sounding methodology, referred to as AIRS Version 5 AO, was developed as a backup to AIRS Version 5 should the AMSU-A instrument fail. Results of Version 5 AO were shown to be almost as good as those of the complete Version 5 AIRS/AMSU processing system. ${ }^{4}$

This paper will concentrate on the accuracy of Version 5 error estimates and their use for quality control in the complete Version 5 AIRS/AMSU processing system. In particular, the spatial coverage and accuracy of retrievals using different 
error estimate thresholds for quality control will be shown, as well as results of forecast impact studies assimilating AIRS Version 5 temperature profiles using different quality control thresholds.

\section{OVERVIEW OF THE AIRS TEAM RETRIEVAL ALGORITHM}

The AIRS team Version 5 and Version 5 AO retrieval algorithms are basically identical to each other and to those described previously ${ }^{2-4}$. The key steps are outlined below: 1) Start with an initial state consistent with the AIRS/AMSU (or AIRS only) observed radiance. 2) Derive IR clear column radiances $\hat{\mathrm{R}}_{\mathrm{i}}^{0}$ valid for the $3 \times 3$ AIRS Fields of View (FOVs) within an AMSU-A Field of Regard (FOR) consistent with the observed radiances and the initial state using 58 AIRS cloud clearing channels; 3) Obtain an AIRS regression guess ${ }^{5}$ consistent with $\hat{R}_{i}^{0}$ using 1504 AIRS channels; 4) Derive $\hat{R}_{i}^{1}$ consistent with the AIRS radiances making use of the regression guess; 5) Derive all surface and atmospheric parameters using $\hat{\mathrm{R}}_{\mathrm{i}}^{1}$ for 308 AIRS channels and AMSU radiances (Version 5 AO does not use the AMSU observations); 6) Derive an improved set of clear column radiances $\hat{R}_{i}^{2}$ using the AIRS physically retrieved parameters; 7) Repeat Step 5 using $\hat{\mathrm{R}}_{\mathrm{i}}^{2}$ to produce the final retrieval state; 8) Derive cloud parameters and OLR consistent with the solution and observed $\mathrm{R}_{\mathrm{i}}$;9) Apply initial quality control, which rejects the final solution if the retrieved cloud fraction is greater than $90 \%$ or other relatively coarse tests fail. In the event that a retrieval is rejected, cloud parameters are determined consistent with the state used for initial cloud clearing, in conjunction with the observed AIRS radiances. Otherwise, cloud parameters are computed using the final retrieval and observed AIRS radiances, and further quality control is applied to individual geophysical parameters.

The major differences between the Version 5 and Version 4 algorithms are related to the new ability to perform cloud clearing using only AIRS observations and the new methodology to determine accurate case-by-case, parameter-byparameter, error estimates. Version 5 and Version 5 AO are otherwise identical except that Version 5 AO does not use AMSU A radiances in any step, including the generation of error estimates and quality control.

\section{VERSION 5 ERROR ESTIMATES AND QUALITY CONTROL}

Coupled AIRS/AMSU-A (or AIRS only) retrievals in the presence of broken cloud cover are usually highly accurate. Under some conditions, such as complete overcast, combined AIRS/AMSU-A retrievals cannot be performed at all. In cases of complex clouds or terrain, retrievals are of poorer quality. In the pre-launch version of the AIRS/AMSU-A retrieval algorithm, quality control was applied uniformly to the entire profile. If any geophysical parameter was considered to be of poor quality, the whole set of retrieval geophysical parameters was rejected and clouds were derived using the MW state of Step (1) above. This "one size fits all" approach led to significant compromises between desired spatial coverage of accepted retrievals and desired accuracy. In Version $4^{3}$, the combined IR/MW retrieval parameters are retained, and used to derive cloud parameters, as long as it is felt that the combined IR/MW retrieval (Step 7) is at least as accurate as the MW only retrieval (Step 1). This was considered to be true if the retrieved cloud fraction derived using the IR/MW state was less than or equal to $90 \%$ and the initial cloud clearing step was stable. If this test was passed (referred to as the Stratospheric Temperature Test), the temperature profile above $200 \mathrm{mb}$ was considered acceptable. Constituent profiles $\left(\mathrm{H}_{2} \mathrm{O}, \mathrm{O}_{3}, \mathrm{CO}\right.$, and $\left.\mathrm{CH}_{4}\right)$ were accepted if the Stratospheric Temperature Test was passed and additional slightly more stringent cloud clearing stability tests were also passed. The next level of test was applied to the temperature profile beneath $200 \mathrm{mb}$ and above $3 \mathrm{~km}$ (the Mid Tropospheric Temperature Test). Finally, a more stringent test was applied to accept temperature profiles in the lowest $3 \mathrm{~km}$ of the atmosphere (Lower Tropospheric Temperature Test). Lower tropospheric temperatures are the most difficult to determine accurately, both because of effects of low clouds on the radiances and uncertainty and small scale variability in surface skin temperature and emissivity. Both concerns create greater problems over land than ocean. In response to this, the Lower Tropospheric Temperature Test rejected lower tropospheric temperature more often over land than over ocean in Version 4.

The Version 4 quality control tests each used thresholds for values of 12 different parameters $Y_{k}(k=1,12)$ representative of residuals of internal convergence tests. ${ }^{3}$ In Version 5, the case-by-case values of each of the parameters whose 
thresholds were used in the Version 4 acceptance tests, $Y_{k}$, are used in the generation of error estimates of the individual retrieved parameters. Values of four other retrieval convergence tests are included as well. In the case of either $\mathrm{T}(\mathrm{p}), \mathrm{T}_{\text {skin }}$, or $\mathrm{W}_{\text {tot }}$ (total precipitable water), we write

$$
\delta \mathrm{X}_{\mathrm{i}}=\sum_{\mathrm{k}=1}^{\mathrm{N}} \mathrm{M}_{\mathrm{ik}} \mathrm{Y}_{\mathrm{k}}
$$

where $\delta \mathrm{X}_{\mathrm{i}}$ is the error estimate of parameter $\mathrm{X}_{\mathrm{i}}, \mathrm{Y}_{\mathrm{k}}$ is the value of the $\mathrm{k}^{\text {th }}$ test, $\mathrm{M}$ is a matrix with different values over ocean and land, and $\mathrm{N}$ is the number of tests used to determine the error estimate. Error estimates are, by definition, all positive. Three of the tests include AMSU-A observations. These tests are not used in Version 5 AO. Therefore, $\mathrm{N}=16$ in Version 5 and $\mathrm{N}=13$ in Version $5 \mathrm{AO}$. Other than the number of tests used, error estimates are computed and used in an analogous manner in Version 5 and Version $5 \mathrm{AO}$.

If one knows the actual errors, given by $X_{i}-X_{i}^{\text {truth }}$, the matrix $M$ is determined in a straightforward manner, by finding $M$ such that $M$ minimizes the RMS difference of $\left(\Delta X_{i}-\delta X_{i}\right)$, where $\Delta X_{i}=\left|X_{i}-X_{i}^{\text {truth }}\right|$. In order to generate $M$, we used $X_{i}$ and $Y_{k}$ for all accepted Version 5 retrievals (that is all cases passing the Version 4 Stratospheric Temperature Test) on September 29, 2004, and used the colocated ECMWF 3 hour forecast used as truth. The set of accepted cases used for training is not the same for Version 5 and Version 5 AO, nor are the resulting coefficients. The coefficients $\mathrm{M}$ for each version are used once and for all.

\subsubsection{Temperature profile quality control}

Case-by-case level-by-level error estimates for temperature profiles are obtained by equation 1 using the appropriate values of $Y_{k}$ for each profile. These error estimates are used to determine a case-by-case characteristic pressure $p_{\text {good }}$, down to which the profile is considered acceptable. All accepted profiles are assigned to have high quality down to at least $70 \mathrm{mb}$. The characteristic pressure $\mathrm{p}_{\mathrm{good}}$ is defined as the highest pressure (somewhere between $70 \mathrm{mb}$ and $\mathrm{p}_{\text {surf }}$ ) at which the error estimate in each of the next 3 pressure levels is not greater than a pressure dependent error estimate threshold. These pressure dependent thresholds vary between $2.25 \mathrm{~K}$ and $1.25 \mathrm{~K}$ throughout the atmosphere, and were optimized separately for Version 5 and Version 5 AO, bearing in mind what was considered to be the best trade-off between accuracy and spatial coverage for use in both data-assimilation and climate applications.

Pressure dependent thresholds are determined from a set of 3 threshold parameters $\delta \mathrm{T}_{70}, \delta \mathrm{T}_{\text {mid }}$, and $\delta \mathrm{T}_{\text {surf }}$, representative of error thresholds for $T(p)$ at $p=70 \mathrm{mb}$, at $\mathrm{p}=\mathrm{p}_{\text {surf } / 2}$, and at $\mathrm{p}=\mathrm{p}_{\text {surf }}$ where $\mathrm{p}_{\text {surf }}$ is the surface pressure. The thresholds $\delta T(p)$ at intermediate pressures are linearly interpolated in log p between the given values. We have found it advantageous to have separate error thresholds for non-frozen ocean on the one hand, and land and ice on the other. Table 1 shows the standard Version 5 thresholds for both non-frozen ocean (called ocean) and other than nonfrozen ocean (called land). Also shown are values of two other sets of thresholds that we have used for experiments. These are more stringent sets of thresholds and are labeled Medium and Tight. 
Table 1

Temperature Profile Thresholds (K)

\begin{tabular}{lcccccc} 
& \multicolumn{3}{c}{ Ocean } & \multicolumn{4}{c}{ Land } \\
& $\delta \mathrm{T}_{70}$ & $\delta \mathrm{T}_{\text {mid }}$ & $\delta \mathrm{T}_{\text {surf }}$ & $\delta \mathrm{T}_{70}$ & $\delta \mathrm{T}_{\text {mid }}$ & $\delta \mathrm{T}_{\text {surf }}$ \\
Standard & 1.75 & 1.25 & 2.25 & 2.25 & 2.0 & 2.0 \\
Medium & 1.75 & 1.0 & 1.75 & 1.75 & 1.0 & 2.0 \\
Tight & 1.75 & 0.75 & 1.75 & 1.75 & 0.75 & 1.75
\end{tabular}

Figure 1 shows an example of temperature profile error estimates and their use for quality control. Figure 1a shows the differences of retrieved $300 \mathrm{mb}$ temperatures from ECMWF "truth" for all accepted ascending orbit Version 5 in January 25, 2003. Gray means missing data. This can be a result of orbit gaps, a missing granule (over central Africa), or (generally very cloudy) areas where successful retrievals were not performed (such as off the northwest coast of the U.S.). The area weighted global mean of the error without quality control is $-0.15 \mathrm{~K}$, and its spatial standard deviation is $1.48 \mathrm{~K}$. Figure $1 \mathrm{~b}$ shows the predicted errors, and Figure $1 \mathrm{~d}$ shows the differences between the predicted error and the absolute value of the actual error. The spatial correlation is 0.52 , and the spatial standard deviation of the error in the prediction is $0.87 \mathrm{~K}$, showing reasonable skill between the actual "error" (which may itself be incorrect due to errors in the truth), and the predicted error. Figure 1c shows the $300 \mathrm{mb}$ error of the quality controlled cases, i.e., cases in which $\mathrm{p}_{\text {good }} \geq 300 \mathrm{mb}$ using (standard) Version 5 quality control thresholds. The spatial distribution of accepted cases is quite extensive, and the standard deviation of the errors for accepted cases has dropped to 1.18K. The largest "errors" for the accepted cases occur over Antarctica, Greenland, and Northern Siberia, in locations where (by definition), the error estimates are low. These are regions in which the ECMWF "truth" may be of poorer quality and actual errors may be less than the errors shown in Figure 1c.

Figure $2 \mathrm{a}$ shows the field of accepted $300 \mathrm{mb}$ temperature with Version 5 standard quality control. The cases shown are identical to those whose errors are shown in Figure 1c. In addition to having very good spatial coverage, the thermal features are well defined and well represented. This good spatial coverage is needed for the purpose of generating climate data sets with a minimal clear sky bias. Very good spatial coverage is also potentially desirable for data assimilation purposes, provided the accuracy of the accepted retrievals is acceptable. Figure $2 b$ shows the spatial coverage of

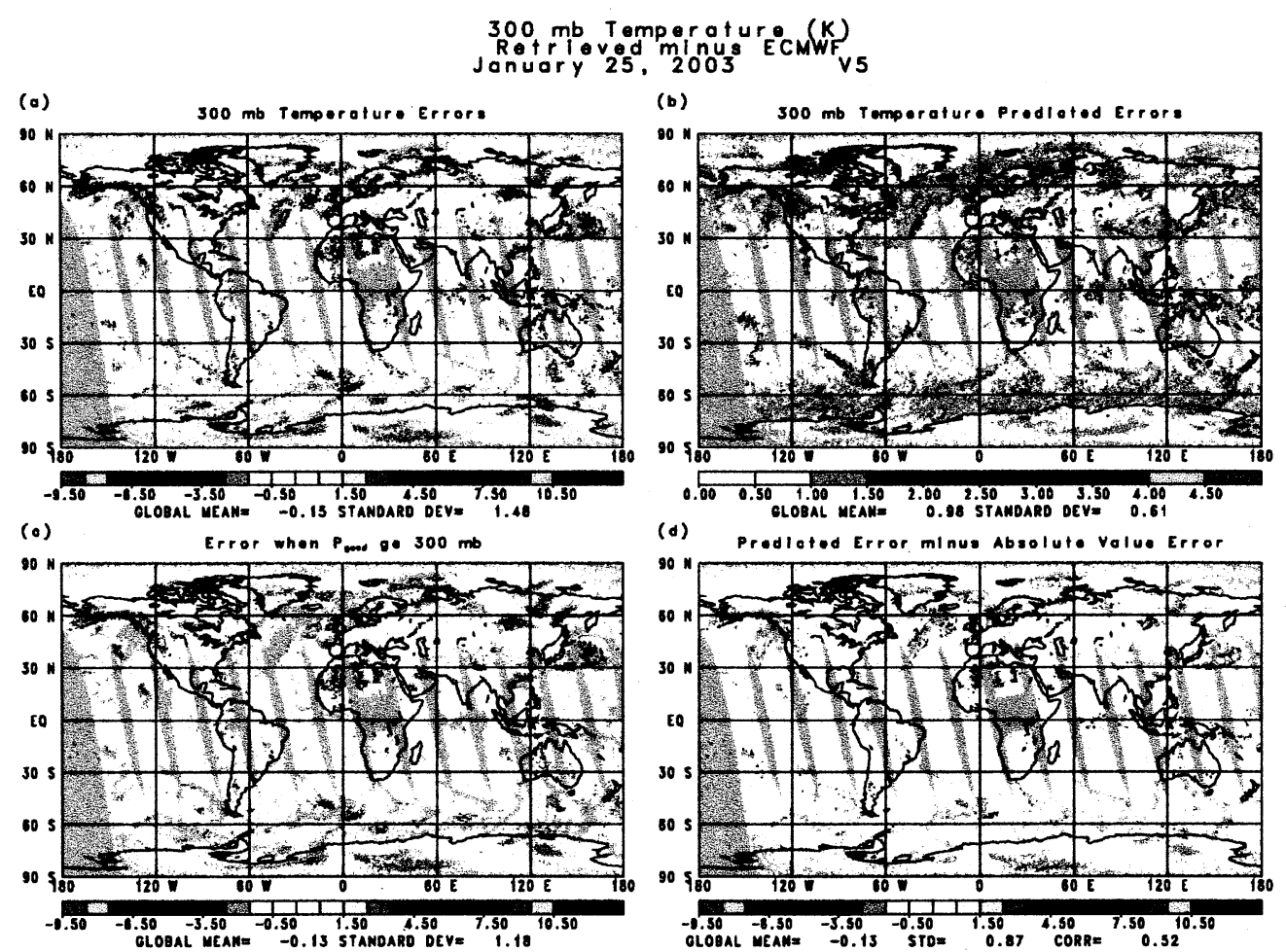

Figure 1 
accepted Version 4 retrievals at $300 \mathrm{mb}$. The Version $4300 \mathrm{mb}$ spatial coverage is reasonably good, but significantly poorer than Version 5 Standard, particularly at high latitudes. Figures 2c and $2 \mathrm{~d}$ show spatial coverage for Version 5 with medium and tight thresholds. Version 5 Medium has somewhat better spatial coverage than Version 4, and Version 5 Tight has poorer spatial coverage than Version 4 at $300 \mathrm{mb}$.

Figure $3 \mathrm{a}$ shows the rms error of global quality controlled temperature profiles for Version 4 (black) and Version 5 (gray) using the standard Version 5 thresholds $\delta \mathrm{T}_{70}, \delta \mathrm{T}_{\text {mid }}$, and $\delta \mathrm{T}_{\text {surf for }}$ both land and ocean. Figure $3 b$ shows the percent of cases accepted for both Version 4 and for Version 5 using the standard cutoffs. The global percent accepted cases for Version 5 Standard is significantly higher than that of Version 4 at $300 \quad \mathrm{mb}$ with a comparable accuracy. The same is true at all levels of the troposphere. The percent accepted above 200 $\mathrm{mb}$ is somewhat lower in Version 5 Standard than in Version 4, with a significant improvement in sounding accuracy between $100 \mathrm{mb}$ and $200 \mathrm{mb}$. This is because not all cases called "stratosphere good" in Version 4 have sufficient accuracy down to $200 \mathrm{mb}$. The red and purple curves represent quality controlled Version 5 retrievals using tighter cutoffs as shown in the figure. They show how tightening thresholds can lead to significantly more accurate quality controlled retrievals, but with a lower percentage of accepted retrievals, resulting in poorer spatial coverage.
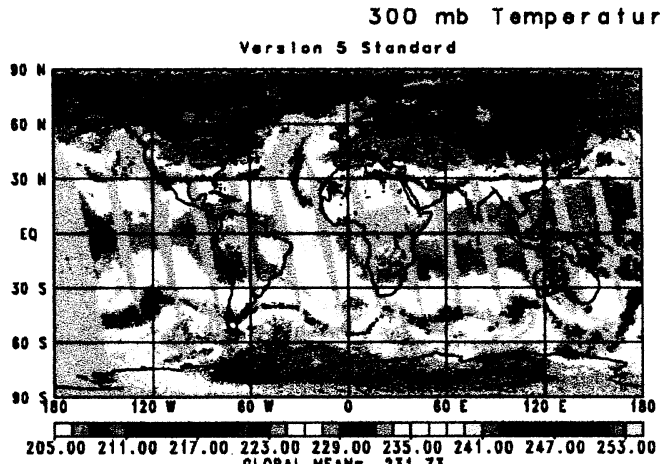

Vorsion 5 Modlum

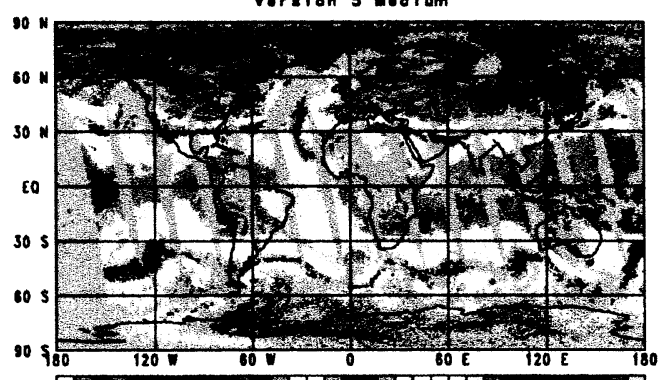

205.00211 .00217 .00223 .00229 .00235 .00241 .00247 .00253 .00

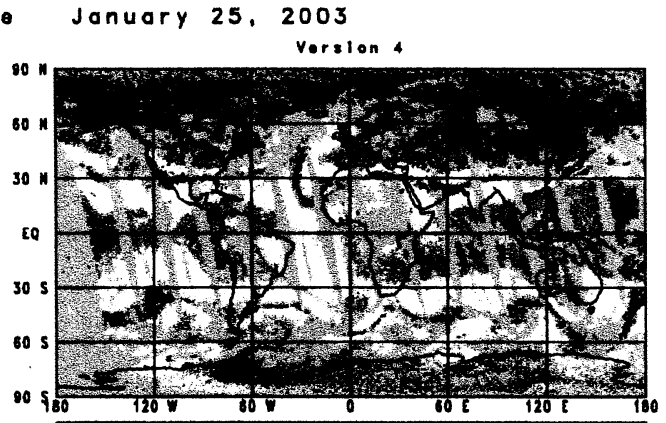

205.00211 .00217 .00223 .00229 .00255 .00241 .00247 .00253 .00

version 5 Tight

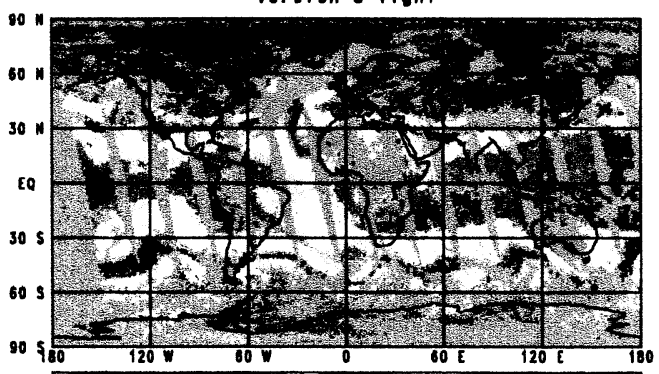

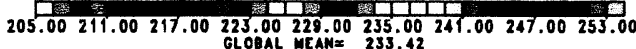

Figure 2
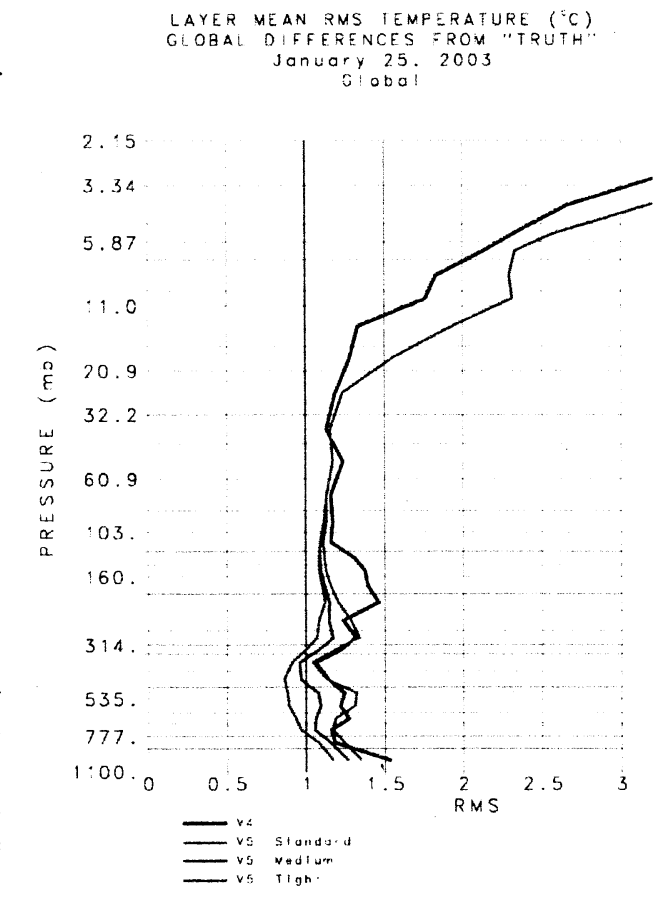

Figure 3

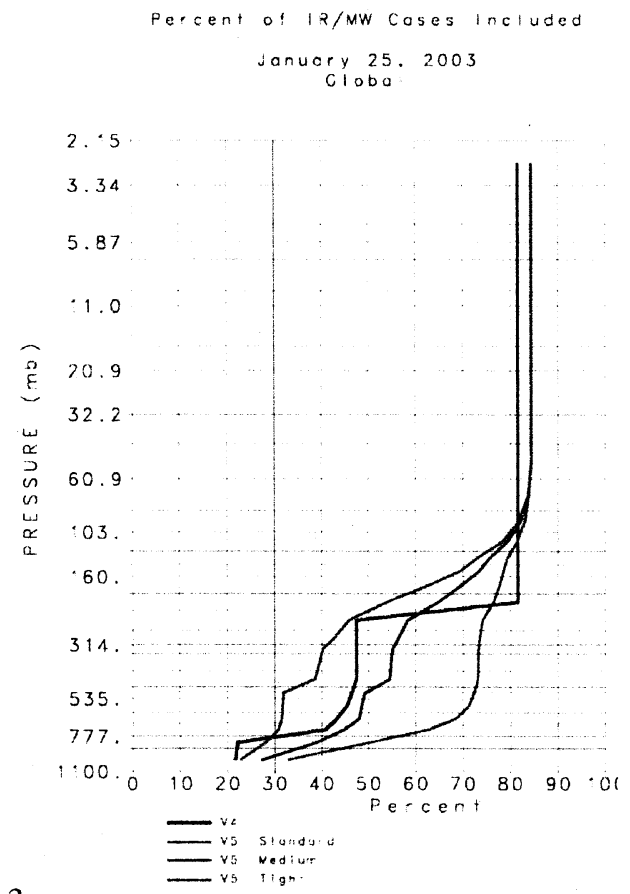


Figure 4 is analogous to Figure 2, but for quality controlled temperatures at 700 mb. Areas with surface pressure less than $700 \mathrm{mb}$, such as over East-Antarctica, show up as data gaps in this figure. The Version 4 spatial coverage over land and extratropical oceans at $700 \mathrm{mb}$ is extremely low. Version 5 Standard has extensive $700 \mathrm{mb}$ spatial coverage globally. Version 5 Tight has spatial coverage at 700 $\mathrm{mb}$ closer to that of Version 4, and Version 5 Medium has intermediate spatial

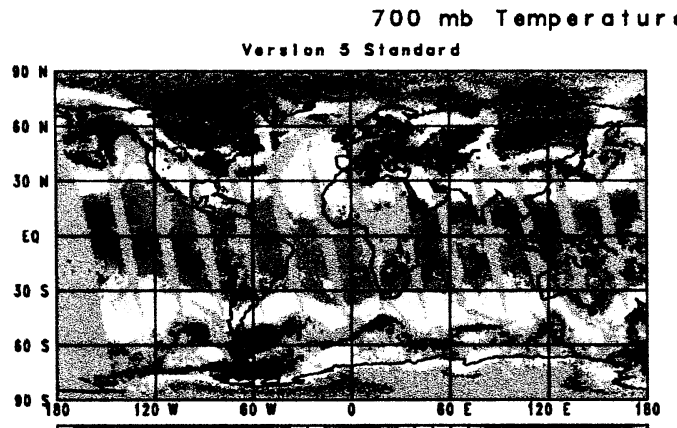
221.00231 .00241 .00251 .00251 .00271 .00281 .00291 .00301 .00 version 5 Modium

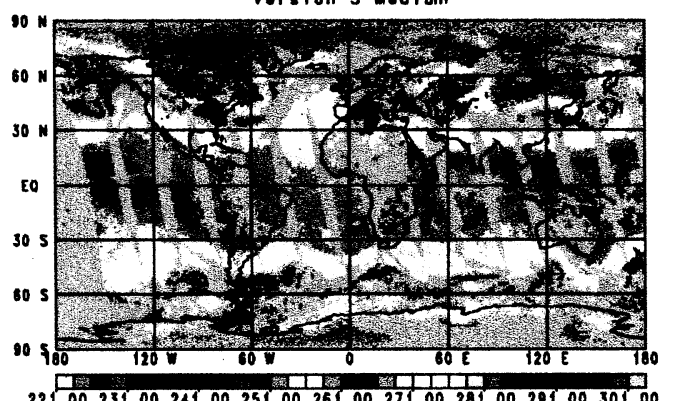
221.00231 .00241 .00251 .00261 .00271 .00281 .00281 .00301 .00
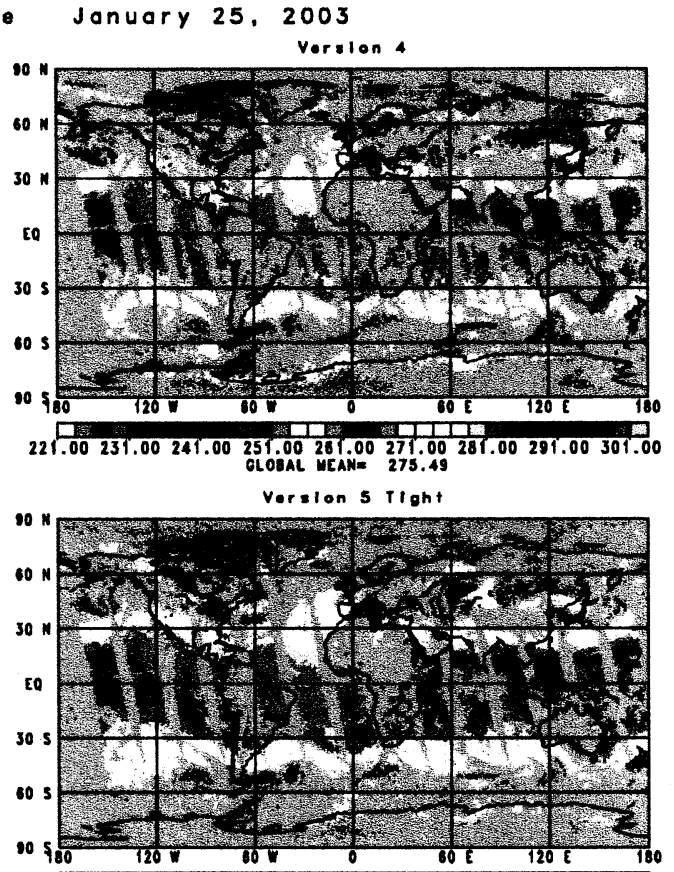

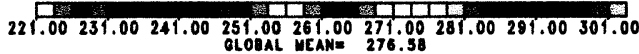
coverage.

Figure 4

\section{FORECAST IMPACT EXPERIMENTS}

We have conducted a number of data assimilation experiments as a step toward finding an optimum balance of spatial coverage and sounding accuracy with regard to improving forecast skill. The data assimilation system used in the experiments is FVSSI which represents a combination of the NASA Finite Volume General Circulation Model $\left(\right.$ FVGCM) ${ }^{6}$ with the NCEP operational Grid Point Statistical Interpolation (GSI) global analysis scheme implemented at lower than the operational horizontal resolution. The GSI is expected to become operational at NCEP in 2007. The basics of the finite-volume dynamical core formulation are given in DAO's Algorithm Theoretical Basis Document (see http://polar.gsfc.nasa.gov/sci research/atbd.php), and the FVGCM has been shown to produce very accurate weather forecasts when run at high resolution. ${ }^{6}$

A number of experiments utilizing AIRS data were conducted. In all experiments, data was assimilated for the period January 1 - January 31,2003 . Five day forecasts were run every two days beginning January 6, 2003 and forecasts every 12 hours were verified against the NCEP analysis, which was taken as "truth".

The first set of experiments assimilated quality controlled AIRS Version 5 retrieved temperatures down to the surface using the three different quality control thresholds described in Section 2. The objective of this set of experiments was to compare the relative forecast skill of assimilating AIRS retrievals with different spatial coverage and error characteristics. The AIRS Version 5 temperature profiles were presented to the GSI analysis as rawinsonde profiles. The predicted AIRS temperature observational errors were used in the generation of the error weights used in the data assimilation process.

Forecasts generated from these three sets of analyses are compared to those from the "Control" analysis, in which all the data used operationally by NCEP was assimilated, but no AIRS data was assimilated. The operational data included all conventional data, TOVS and ATOVS radiances for NOAA-14, 15, and 16, cloud tracked winds, SSM/I total 
precipitable water and surface wind speed over ocean, QuikScat surface wind speed and direction, and SBUV ozone profiles. Radiances from Aqua AMSU instrument were also assimilated operationally by NCEP and are included in the "Control". No AIRS data was assimilated operationally at that time.

Figure 5 shows the average of the 2712 hour to 5 day forecast sea level pressure anomaly correlation coefficients verified against the NCEP analysis for both Northern Hemisphere extratropics and Southern Hemisphere extra-tropics for all these experiments. In the Northern Hemisphere, assimilating AIRS soundings with error weights resulted in an improvement in average 5 day forecast skill varying from 2 hours (Tight) to 4 hours (Medium and Standard). The improvement in average 5 day forecast skill in the Southern Hemisphere extra-tropics varied from 3 to 6 hours.

The differences in forecast skill using different Quality Control thresholds was larger in the Southern Hemisphere Extra-tropics than in the Northern Hemisphere Extratropics. Medium Quality Control produced somewhat better forecasts than the Standard Quality Control. The Tight Quality Control produced the smallest, but still significant, positive impact resulting from assimilation of AIRS temperature soundings. This implies that there should be an optimum balance between spatial coverage and overall accuracy for maximum forecast impact. The Tight Quality Control did produce the best overall retrieval accuracy, but had sub-optimal spatial coverage for data assimilation purposes.
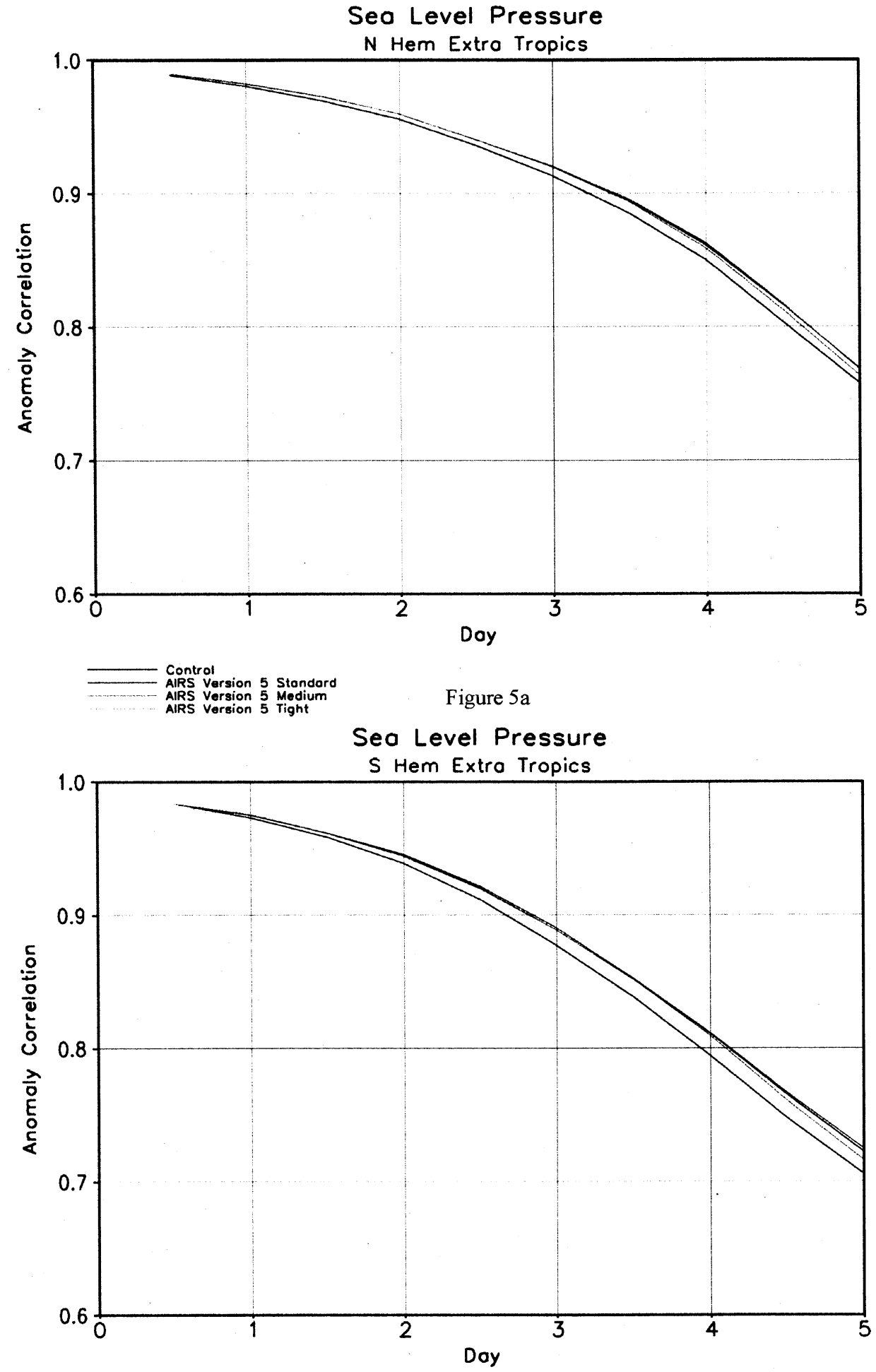

Control AIRS Version 5 Standord AIRS Version 5 Medium
Figure 5b 
This set of experiments shows that assimilation of Quality Controlled AIRS temperature soundings extending down to temperatures at the surface air pressure significantly improves forecast skill in both the Northern and Southern Hemisphere extra-tropics. Assimilation of soundings with Medium Quality Control performed slightly better than with Standard Quality Control, and significantly better than with Tight Quality Control. It should be noted that the Aqua orbit (1:30 ascending) is almost identical to that of NOAA 16 carrying HIRS3, AMSU A and AMSU B, so AIRS/AMSU temperature soundings are providing additional information to that contained in the AMSU A/AMSU B radiances on NOAA 16 in the same orbit, as well as those of the Aqua AMSU radiances themselves.

$\mathrm{NCEP}^{7}$ and ECMWF now assimilate AIRS observations operationally. The current operational practice at both Centers is to directly assimilate observed AIRS radiances rather than AIRS temperature soundings as done in the first set of experiments. Successful AIRS temperature soundings are generated under almost all cloud conditions (see Figures 2 and 4 for examples of the spatial coverage of accepted retrievals at $300 \mathrm{mb}$ and $700 \mathrm{mb}$ ) using cloud cleared radiances. The ability to derive and use accurate cloud cleared radiances is a critical element in the optimal use of AIRS data for both data assimilation and climate purposes.

The operational methodologies used by both NCEP and ECMWF do not have the capability to derive and assimilate cloud cleared AIRS radiances. Instead, the analysis procedures used at both Centers select and assimilate only these AIRS observations which are "thought to be unaffected by clouds." These uncontaminated radiance observations are primarily in the stratosphere and also above clouds in areas where clouds are present. Our results from AIRS indicate that roughly $95 \%$ of AIRS pixels are cloud contaminated. Therefore, most tropospheric sounding AIRS observations are not included in the operational AIRS radiance assimilation process.

The second set of experiments which we conducted were designed to assess the relative importance of assimilating only AIRS Quality Controlled temperature soundings in the Stratosphere compared to assimilating Quality Controlled AIRS temperature soundings down to the surface. In this experiment, we used the AIRS Medium Quality Control as described above, but only assimilated soundings down to at most $200 \mathrm{mb}$. For comparison, we also assimilated AIRS radiances according to the NCEP operational procedure. As discussed above, this also provides mostly stratospheric information from AIRS to be used in the data assimilation process. In the AIRS Radiance Assimilation experiments, all other data assimilated in the control was also included, but no AIRS temperature profile data was assimilated.

Figure 6 shows analogous results to those shown in Figure 5, but for Northern Hemisphere and Southern Hemisphere Extra-Tropics $500 \mathrm{mb}$ Geopotential Height anomaly coefficients. Results are shown for forecasts using the control (black) and AIRS Version 5 Medium (red) analyses as before. Also shown are results from the AIRS Version 5 Medium down to $200 \mathrm{mb}$ (orange) and AIRS Radiance Assimilation Analyses (green). Figure 6 shows the very significant result that virtually all the positive impact of assimilation of Quality Controlled AIRS temperature soundings is lost if only AIRS stratospheric temperatures are used in the assimilation process (orange). The most important information is coming from tropospheric temperatures which are determined and assimilated primarily in partially cloudy scenes. Assimilation of AIRS radiances unaffected by clouds results in essentially no forecast impact in the Northern Hemisphere Extra-Tropics, and a significantly reduced positive forecast impact in the Southern Hemisphere Extratropics compared to the assimilation of AIRS Quality Controlled temperature profiles down to the surface. At least a part of this reduction in forecast impact of radiance assimilation compared to temperature assimilations results from the significant loss of spatial coverage in AIRS tropospheric sounding channels due to cloud contamination. 


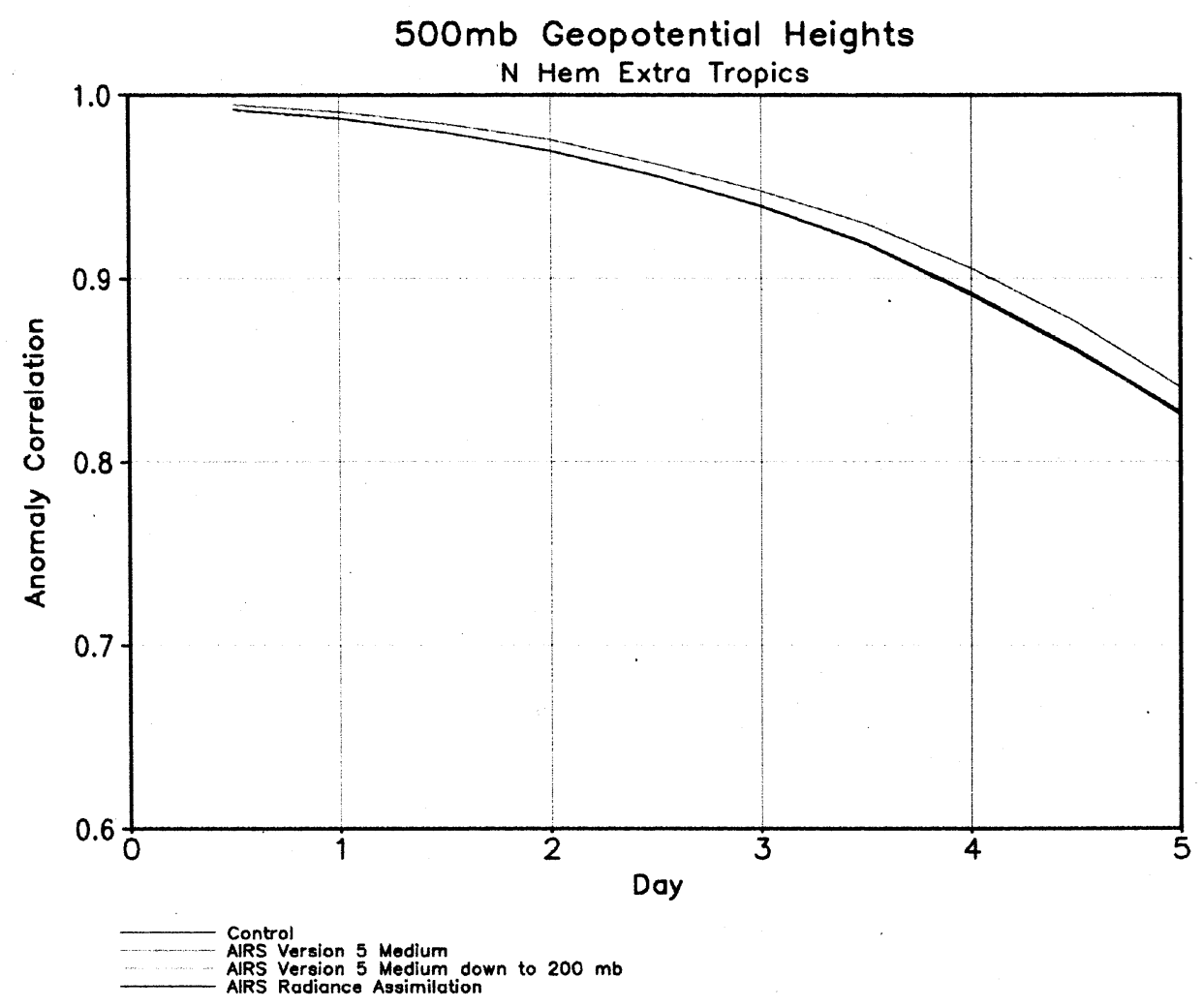

Figure 6a

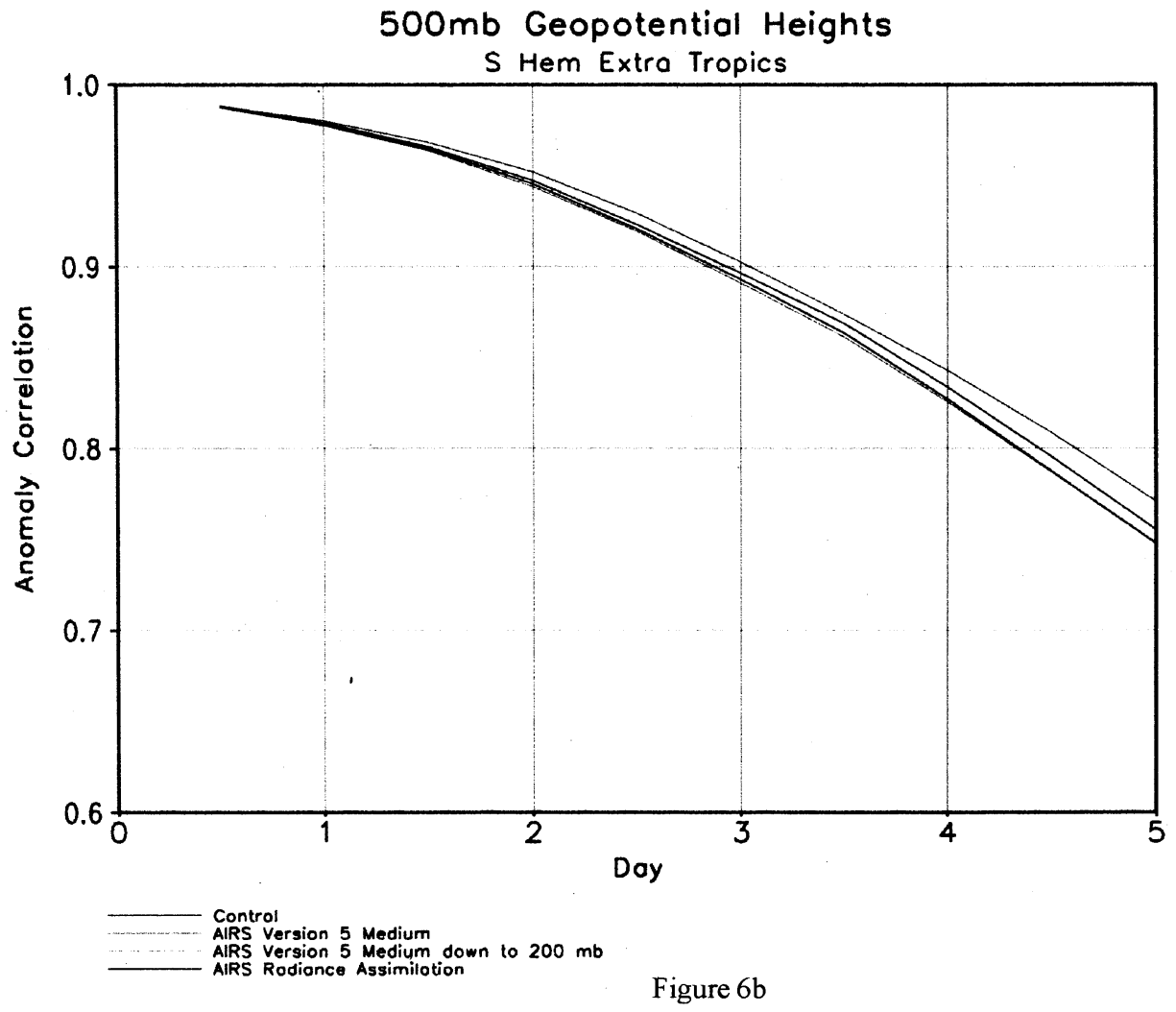


The results shown in Figures 5 and 6 represent averages of anomaly correlation coefficients for each of the 27 forecasts in the ensemble. It is very informative to look at the skill of each of the 27 forecasts in the ensemble. Figure 7 shows 5 day forecast anomaly correlation coefficients for each of the 27 days in the ensemble for each of the four experiments included in Figure 6 . In the Northern Hemisphere ExtraTropics, 5 day forecasts from the Control experiment (black) were poor on days 4, 8, and 17 . The assimilation of complete Medium Quality Controlled AIRS soundings (red) significantly improved 5 day forecast skill on these days and also improved on skillful Control forecasts in days 21-27. Days 6, 12, and

19 had very skillful 5 day forecasts from the Control that were degraded slightly in the full AIRS temperature profile data assimilation system, but these "degraded forecasts" were still quite skillful, with anomaly correlation coefficients greater than 0.8. Skill scores of the forecasts resulting from assimilation of AIRS stratospheric temperatures (orange) and AIRS radiances (green) are much closer to those from the control on almost a case-by-case basis. The 5 day forecast from the radiance assimilation does improve on the very poor forecast from the Control

on day 8 , but much less so than the improvement resulting from the full AIRS temperature profile assimilation.

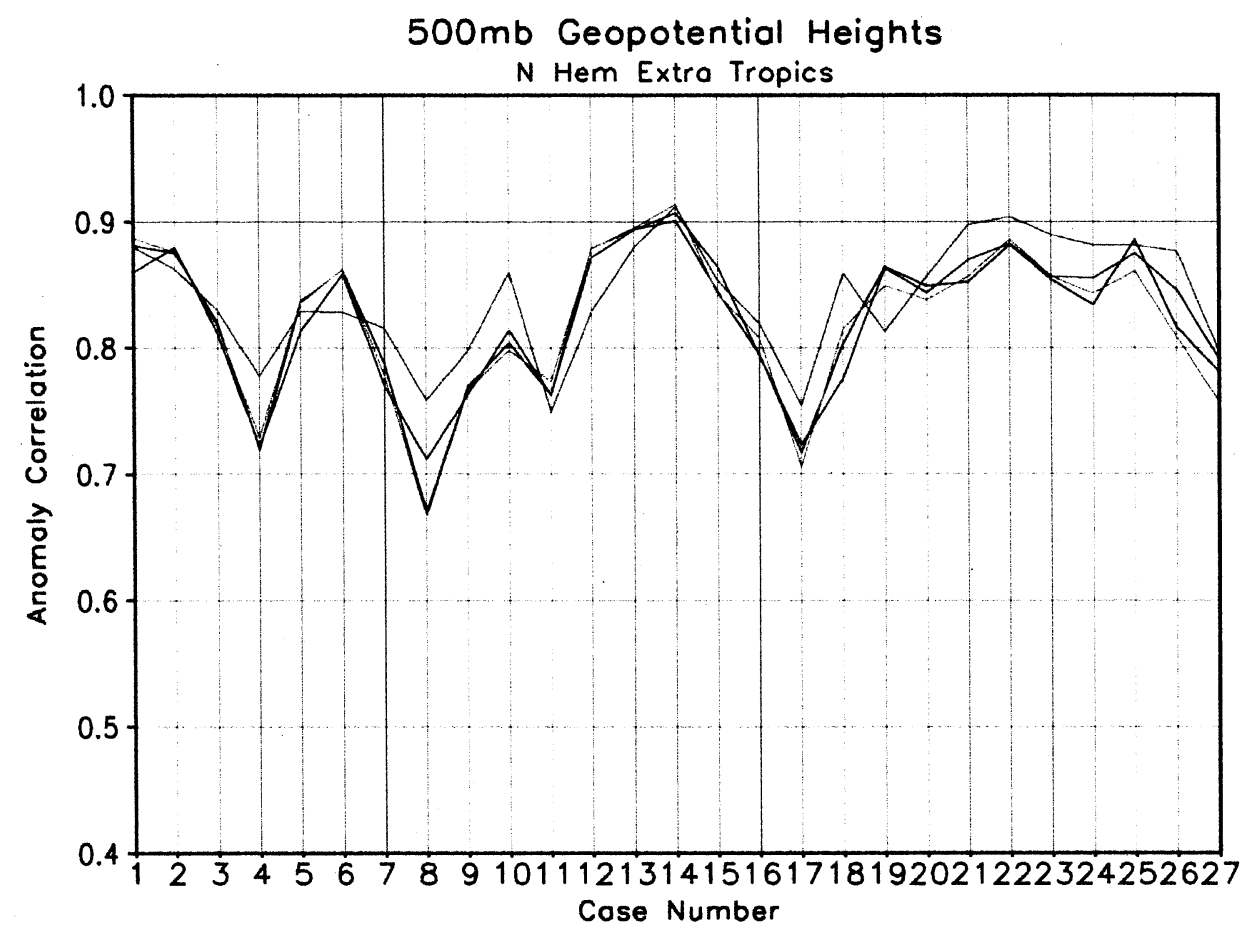

Figure 7a

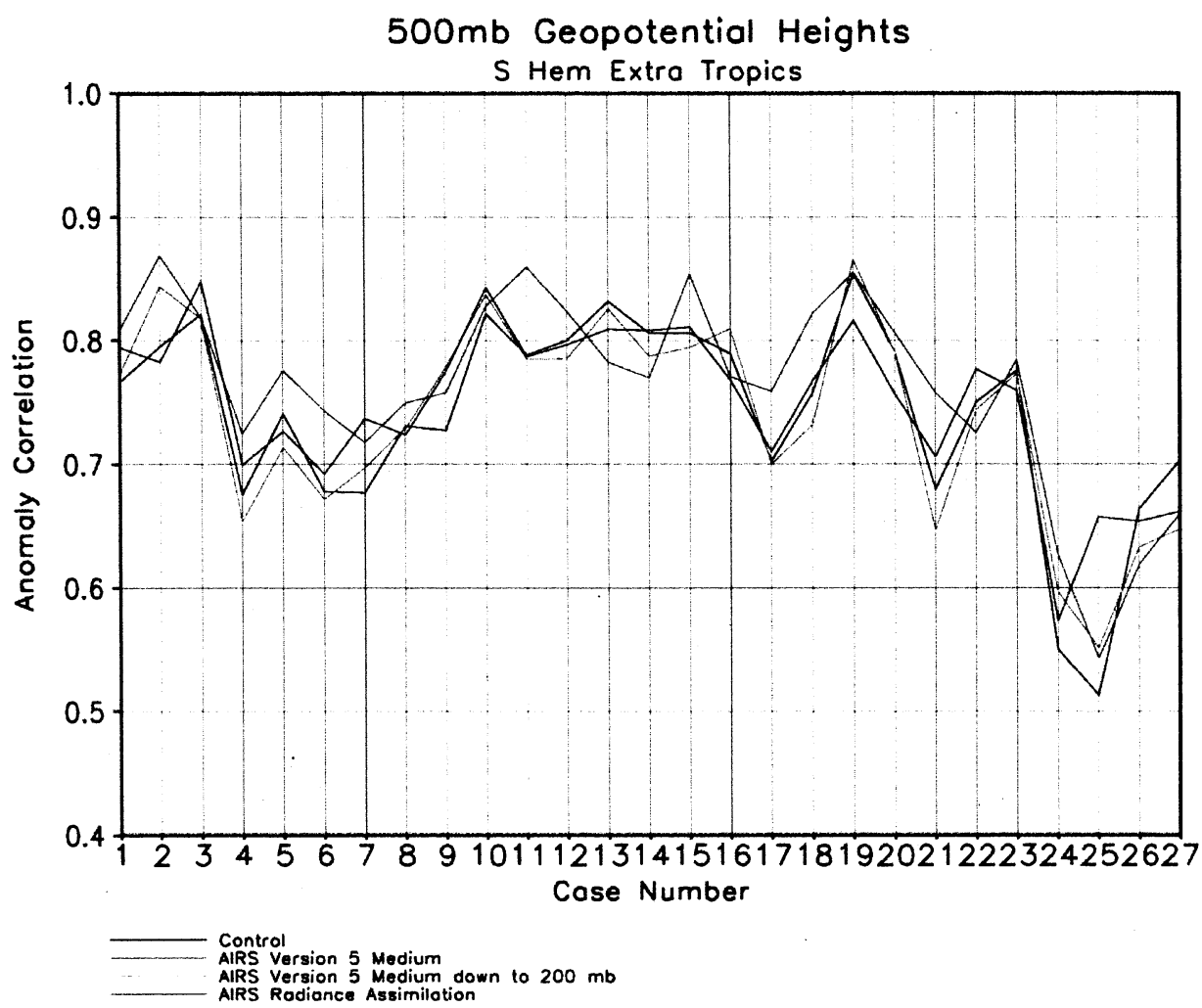

Figure $7 b$ 
General findings with regard to individual 5 day Southern Hemisphere extra-tropics forecasts are similar to those in the Northern Hemisphere extra-tropics. All 5 day forecasts in the Southern Hemisphere extra-tropics from day 25 have marginal or very poor skill. On this day, the forecast from the radiance assimilation is not as poor as the others however.

\section{SUMMARY}

The AIRS Science Team Version 5 retrieval algorithm has been finalized and is now operational at the Goddard DAAC in the processing (and reprocessing) of all AIRS data. Version 5 contains accurate case-by-case (and channel by channel) error estimates for most derived products, which are also used for quality control. Assimilation of these quality controlled temperature profiles resulted in significantly improved forecast skill in both the Northern Hemisphere and Southern Hemisphere Extra-Tropics. Experiments using different Quality Control thresholds for assimilation of AIRS temperature retrievals showed that an optimal balance should exist between sounding accuracy and sounding spatial coverage for best use in data assimilation. We are conducting more experiments to further optimize this balance from the data assimilation perspective.

In all cases, soundings were assimilated well below cloud level in partially cloudy cases. The positive impact of assimilating AIRS derived atmospheric temperatures all but vanished when only AIRS stratospheric temperatures were assimilated. Forecast skill resulting from assimilation of AIRS radiances uncontaminated by clouds was only slightly better than that resulting from assimilation of only stratospheric AIRS temperatures. This reduction in forecast skill is most like the results of significant loss of tropospheric information when only AIRS radiances unaffected by clouds are used in the data assimilation process.

\section{REFERENCES}

1. T. S., Pagano, H. H. Aumann, D. E. Hagan, and K. Overoye, "Prelaunch and in-flight radiometric calibration of the Atmospheric Infrared Sounder (AIRS)," IEEE Trans. Geosci. Remote Sensing, 41, 265-273, 2003.

2. J. Susskind, C. D. Barnet, and J. M. Blaisdell, "Retrieval of atmospheric and surface parameters from AIRS/AMSU/HSB data in the presence of clouds," IEEE Trans. Geosci. Remote Sensing, 41, 390-409, 2003.

3. J. Susskind, C. Barnet, J. Blaisdell, L. Iredell, F. Keita, L. Kouvaris, G. Molnar, and M. Chahine, "Accuracy of geophysical parameters derived from Atmospheric Infrared Sounder/Advanced Microwave Sounding Unit as a function of fractional cloud cover," J. Geophys. Res., 111,D09S17, doi:10.1029/2005JD006272, 2006.

4. J. Susskind, "Improved soundings and error estimates using AIRS/AMSU-A data," edited by Sylvia S. Shen, Paul E. Lewis, Proc. of SPIE, 6233, 623319-1-623319-12, 2006.

5. J. Susskind, "Recent theoretical advances in analysis of AIRS/AMSU sounding data," Proc. of SPIE Vol. 6565, Algorithms and Technologies for Multispectral, Hyperspectral, and Ultraspectral Imagery XIII, Orlando, FL, April 9-12, 2007, 65651H, 0277-786X/07/\$18,doi:10.1117/12.718448, 65651H-1 - 65651H-12, 2007.

6. S. J. Lin, R. Atlas, and K. S. Yeh, "January-February, Global weather prediction and high end computing in NASA," Computing in Science and Engineering, 29-35, 2004.

7. J. Marshall, L. Uccellini, F. Einaudi, M. Colton, S. Chang, F. Weng, M. Uhart, S. Lord, L. Riishojgaard, P. Phoebus, and J. Yoe, "The Joint Center for satellite data assimilation," DOI:10.1175, BAMS, 88-3, 329-340, 2007. 